\title{
A comparative study of subjective experiences related to driving among outpatient psychotropic users and controls in Ragama, Sri Lanka
}

\author{
M Chandradasa, L Champika, M Amarasuriya, P Wijelakshman, S Bandara, T Ranaweera, \\ L Fernando
}

\section{Background}

Difficulties in driving may occur due to the effects of psychiatric disorders or psychotropic medications.

Aim

To identify and explore the impact of illness and medications on the driving of patients visiting an outpatient clinic.

\section{Method}

A retrospective-cohort design was used. The data was collected using a semi-structured, intervieweradministered questionnaire among consecutive patients with schizophrenia or bipolar disorder. An age, gender and vehicle matched control group was obtained.

Result

A total of 2887 consecutive clinic patients were asked about driving and 57 (1.9\%) who had driven a vehicle regularly during the last 12 months were included in the study group. A majority were in the 30-45 year category (47\%). All were men and a majority were motor-bike riders (53\%). Collision-accidents were experienced by $42 \%$ of participants during the last 12 months.

\section{Conclusion}

The patient group had lower hours of driving per week, less subjectively claimed earning per day and more with at least one collision-accident during the past year compared to the control group.

Key words: Automobile driving, psychotropic drugs, comparative study.

SL J Psychiatry 2016; 7(1): 23-25

\section{Introduction}

Driving a vehicle may be required as a professional requirement or as part of travelling in day-to-day life. It has become an essential part of life in the 21st century. Inability to drive may reduce earning capacity or hinder a person's ability to fulfil their occupational and social roles.

Difficulties in driving may occur due to psychiatric disorders or treatment given for psychiatric illness (1). Psychotropic medications may affect visual, cognitive and motor skills that are required for safe driving (2). For example, a medication with anticholinergic side effects may cause blurred vision. Antipsychotics could also impair the cognitive abilities needed for driving (2). They are also known to cause extrapyramidal side effects including stiffness and tremor, which might impair the motor skills required for safe driving (3).

Psychiatric disorders such as schizophrenia can affect the cognitive abilities of the person with the illness (4). Perceptual abnormalities including auditory and other hallucinations may be associated with impaired attention, which is essential in safe driving (5). Parkinson's and Huntington's diseases could impair the motor skills relevant for driving $(6,7)$.

Almost all categories of medications acting on the central nervous system have the potential to influence the driving ability of an individual. Evidence shows that anxiolytics, antipsychotics, antidepressants, anticonvulsants and benzodiazepines may all adversely affect the driving capacities of patients prescribed these medications (8-10).

The clinician who prescribes a medication with a potential impact on driving has an obligation to inform the relevant client regarding the possible dangers. This may mean that the patient may have to stop driving for a certain period. There are no clear guidelines for driving for persons with psychiatric illnesses in Sri Lanka. However regulations related to driving state that a person who is suffering from a psychiatric disorder may have to be assessed by a psychiatrist before the licence is issued (11). 
A recent study on Sri Lankan drivers reported a high prevalence of road rage and significant associated psychological distress among them (12). But there are no published studies on drivers with psychiatric disorders treated with psychotropics for Sri Lanka. Since the number of vehicles in the country is rising rapidly, it is likely that more individuals on psychotropics would be driving in the future. The objective of this study was to assess the driving and problems related to driving, in a group of patients suffering from psychiatric disorders who were prescribed psychotropic medications.

\section{Methods}

A retrospective cohort study design was used. The study was conducted at the psychiatry outpatient clinic of the Colombo North Teaching Hospital, Ragama. Patients diagnosed to be suffering from schizophrenia or bipolar affective disorder with psychotic symptoms, according to the 10th edition of International Classification of diseases were included in the study. A convenient sample of adult patients using at least one antipsychotic and who had driven regularly during the last 12 months were invited to participate. Regularity of driving was defined for study purposes as driving a motor vehicle at least once weekly. An age, gender and vehicle matched control group was obtained from the non-patient, non-staff drivers visiting the Ragama hospital.

A semi-structured interviewer-administered questionnaire was used to collect the data. Details regarding driving was obtained from the relevant participant, and clarified with collateral information from a family member in all participants. The details on the diagnosis and medications were gathered from the hospital records. The statistical significance of the associations for mean values was calculated by the independent t-test and for nominal parameters by the chi-square test using the SPSS software. We obtained written informed consent from all the participants and approval of the Ethical review committee of Faculty of Medicine, University of Kelaniya.

\section{Results}

A total of 2887 consecutive clinic patients were asked about driving and 57 (1.9\%) who had driven a vehicle regularly during the last 12 months were included in the study group. The control group also included 57 participants. All drivers were men, as no female patients were driving regularly. A majority were in the age category of $30-45$ years (47\%), followed by $26 \%$ of the participants each in the 45-60 year and 18-30 year age categories respectively. Among the drivers, motor-bike riders were the commonest (53\%) followed by trishaw drivers (37\%) and drivers of cars (5\%).

In the patient group most participants drove on average for 1-5 hours (53\%) per week. Regarding the distance,
$37 \%$ reported having covered 50-100 km and 26\% covered $10-50 \mathrm{~km}$ on average per week. The reported average earning per day via driving was Rs. $500-1000$ in 27\% and Rs. $1000-2000$ in $26 \%$ of participants. Among the participants, schizophrenia was the diagnosis in 53\% and the rest had bipolar affective disorder with psychotic symptoms. Olanzapine was prescribed for $42 \%$ and risperidone in $31 \%$. Clinically detectable extrapyramidal symptoms were seen among $31 \%$ of study group participants. Day-time sedation and blurred vision were reported by $32 \%$ and $11 \%$ of the participating patients respectively. Among the patient group 21\% claimed they had to drive despite difficulties, in order to earn a living. A minority of $11 \%$ believed their driving to be improved with medications.

For study purposes, a collision accident was defined as a situation where the participant's vehicle had collided with another vehicle, pedestrian or property. At least one collision accident was experienced by $42 \%(n=24)$ of the study group compared to $23 \%$ of the control group $(n=13)$ during the last 12 months $(2=4.84, \mathrm{p}=0.03$ at CI 95\%). This difference occurred despite the higher hours of driving reported for the control group compared to the patient group. The mean average hours of driving per week, for the two groups were 5.17 and 8.67 hours respectively ( $\mathrm{t}=-3.89, \mathrm{p}=0.001$ at $\mathrm{CI} 95 \%)$. The cost of damages due to collision accidents ranged from Rs. 2500 to 150,000 in both groups. Compared to the control group there was a statistically significant reduction in the subjectively claimed earning per day in the study group $(\mathrm{t}=-1.97, \mathrm{p}=0.04$ at $\mathrm{CI}$ 95\%).

\section{Discussion}

In a study conducted in the same hospital in 1998 among patients with epilepsy, $24.8 \%$ of the participants were found to be driving a vehicle (13). In contrast, only $1.9 \%$ of persons drove regularly, from amongst the 2887 psychiatric patients screened for this study. Even though the values may not be directly comparable, this suggests the potential difference between psychiatric patients and a group of patients with a chronic medical disorder. Similarly, a study from neighbouring India has shown that negative attitudes towards mental illness, contributes to exclusion from employment (14).

The patient group on psychotropics had a statistically significant lower amount of hours of driving per week and on average, earned less per day compared to the control group. This may be due the effects of illness or medications, or secondary to stigma associated with mental disorders (1-4,14).

More patients compared to controls, had at least one collision accident during the last 12 months, despite driving for a lesser duration. This finding could have been influenced by several confounders, such as the road and vehicle condition and the time of driving. 
Despite this, the higher rate of at least one collision accident among the patients, should alert the clinician regarding potential dangers related to driving. There is a need for formulation of local guidelines on driving for patients with mental disorders, as decisions regarding this important issue should take into account both the patient's rights and safety of the public. The time has arrived for the mental health professionals to undertake this task in collaboration with government policy makers. Finally, the authors propose that the results of this study could serve as a stepping-stone for researchers to use more advanced technology and further study how psychiatric illness and treatment may effect driving in Sri Lanka.

\section{Declaration of interest}

None declared

M Chandradasa, Department of Psychiatry, Faculty of Medicine, University of Kelaniya, Ragama

L Champika, M Amarasuriya, P Wijelakshman, S Bandara, T Ranaweera, L Fernando, Colombo North Teaching Hospital, Ragama

Corresponding author: $\mathrm{M}$ Chandradasa

Email: miyuruc@kln.ac.lk

\section{References}

1. Segmiller FM, Buschert V, Laux G, et al. Driving skills in unmedicated first- and recurrent-episode schizophrenic patients. Eur Arch Psychiatry Clin Neurosci 2015.

2. Gerhard U, Hobi V. Cognitive-psychomotor functions with regard to fitness for driving of psychiatric patients treated with neuroleptics and antidepressants. Neuropsychobiology 1984; 12(1): 39-47.
3. Judd LL. The effect of antipsychotic drugs on driving and driving related psychomotor functions. Accid Anal Prev 1985; 17(4): 319-22.

4. De Nijs J, Zoun K, Investigators G. The association between cognitive deficits and different outcomes of schizophrenia. Psychiatr Pol 2014; 48(6): 1087-104.

5. Loberg EM, Jorgensen HA, Kroken RA, Johnsen E. Auditory verbal hallucinations reflect stable auditory attention deficits: a prospective study. Cogn Neuropsychiatry. 2015; 20(1): 81-94.

6. Crizzle AM, Classen S, Uc EY. Parkinson disease and driving: an evidence-based review. Neurology 2012; 79(20): 2067-74.

7. Devos H, Nieuwboer A, Vandenberghe W, Tant M, De Weerdt W, Uc EY. On-road driving impairments in Huntington disease. Neurology 2014; 82(11): 956-62.

8. Ravera S, van Rein N, de Gier JJ, de Jong-van den Berg LT. Road traffic accidents and psychotropic medication use in the Netherlands: a case-control study. Br J Clin Pharmacol 2011; 72(3): 505-13.

9. Kaussner Y, Kenntner-Mabiala R, Hoffmann S, Klatt J, Tracik F, Krüger HP. Effects of oxcarbazepine and carbamazepine on driving ability: a double-blind, randomized crossover trial with healthy volunteers. Psychopharmacology (Berl) 2010; 210(1): 53-63.

10. Daurat A, Sagaspe P, Motak L, et al. Lorazepam impairs highway driving performance more than heavy alcohol consumption. Accid Anal Prev 2013; 60: 31-4.

11. Guidelines of the National Transport Medical Institute (Internet). Colombo c2016. Available at: www. transport. gov.lk/web/ (accessed Mar 2016).

12. Rodrigo A, Perera D, Eranga VP, Peris MU, Pathmeswaran A. Road rage in Sri Lanka: prevalence and psychiatric distress. Ceylon Med J 2015; 60(3): 86-90.

13. Seneviratne SL, Gunatilake SB, Adhikari AA, De Silva HJ. Driving and epilepsy in Sri Lanka. Seizure 1998; 7(4): 305-8.

14. Trani JF, Bakhshi P, Kuhlberg J, et al. Mental illness, poverty and stigma in India: a case-control study. BMJ Open 2015 23; 5(2). 